CALT-68-2080

\title{
Quantum solitons at strong coupling
}

\author{
I. W. Stewart ${ }^{1,2}$ and P. G. Blunden ${ }^{1,3}$ \\ ${ }^{1}$ Department of Physics, University of Manitoba, Winnipeg, Manitoba, Canada R3T 2N2 \\ 2 Department of Physics, California Institute of Technology, Pasadena, CA 91125 \\ ${ }^{3}$ Institute for Nuclear Theory, University of Washington, Seattle, WA 98195
}

\begin{abstract}
We examine the effect of one loop quantum corrections on the formation of nontopological solitons in a strongly coupled scalar-fermionic Yukawa theory. The exact one fermion loop contribution is incorporated by using a nonlocal method to correct the local derivative expansion approximation (DE) of the effective action. As the Yukawa coupling is increased we find that the nonlocal corrections play an increasingly important role. The corrections cause the scalar field to increase in depth while maintaining its size. This increases the energy of the bag configuration, but this is compensated for by more tightly bound fermionic states with lower energy. In contrast to the semiclassical picture without quantum corrections, the binding energy is small, and the total energy scales directly with the Yukawa coupling. This confirms the qualitative behavior found in earlier work using the second order DE, although the quantitative solutions differ.
\end{abstract}

11.10.Lm, 03.70.+k

Typeset using REVTEX 
In theories that support static nontopological soliton solutions at the semi-classical level, it is of interest to ask what effect quantum corrections may have on the form of the solution. In determining these solutions, one must face the issue of self-consistency — solving a set of coupled nonlinear differential equations to find the field configuration that minimizes the energy functional. This makes it useful to have a local expansion of the effective action to model these vacuum effects. One such local method is to expand the one loop effective action in momentum space about zero momentum. For nontrivial background fields this is an expansion in increasing orders of background field derivatives, the so called derivative expansion (DE). Such an expansion has been considered in theories of finite nuclei [1], QED [2], and Friedberg-Lee type soliton models [3] 5 .

Of considerable interest is whether the DE converges rapidly enough to make it a useful tool. The nature of the convergence has been studied by several authors [6 9]. In a recent study [10], we have devised a method in $3+1$ dimensions that enables the DE contribution to the field equations to be corrected for cases where it does not converge. This allows the one loop fermionic vacuum scalar density to be included exactly. The method relies on noticing that a DE of the fermion Green function $S(\boldsymbol{x}, \boldsymbol{x} ; \omega)$ works well for large loop energies $\omega$ and for higher partial waves. This method involves making a partial wave expansion of the scalar vacuum density, and correcting smaller partial wave DE contributions by an exact evaluation of the fermionic Green function. Details of the method and a discussion of its reliability can be found in [10].

In this article we consider the effect that vacuum corrections can have on the selfconsistent solutions of a soliton model. For simplicity, we consider the nontopological soliton model of Bagger and Naculich [3]. These authors solve for bound states of $N$ fermions with a Yukawa coupling to a dynamic scalar field, while including the effects of the one loop fermion vacuum using the DE to second order in derivatives of the scalar field. However, it is not clear a-priori that the DE is applicable for this model. This is because the DE is an asymptotic expansion in inverse powers of $m R$, where $m$ is the fermion mass and $R$ is typically the soliton size or surface thickness. Thus, it is only appropriate for sufficiently 
large solitons with $R \gg 1 / m$. Hence the applicability of the DE will depend on whether $1 / m$, or some other scale in the Lagrangian, is instrumental in determining the size of the soliton self-consistent solution.

The choice of Lagrangian density is 3

$$
\mathcal{L}=\sum_{i=1}^{N} \bar{\psi}_{i}\left[i \not \partial-\frac{g}{\sqrt{N}} \phi\right] \psi_{i}+\frac{1}{2}\left(\partial_{\mu} \phi\right)^{2}-\frac{\mu^{2}}{8 N v^{2}}\left(\phi^{2}-N v^{2}\right)^{2}
$$

There are $N$ flavors of fermions in this model, and we work in the limit of large $N$. The utility of the large $N$ parameterization is to validate the semi-classical treatment of the scalar field. In particular, the scalar loop contributions are suppressed by a factor $1 / N$. The scalar field has a nonzero vacuum expectation value (VEV), $\phi_{v}=\langle\phi\rangle=\sqrt{N} v$, and the fermion mass is identified as $m=g v$. Note that due to the presence of a Landau pole [3], only models with $g \lesssim 30$ are physically acceptable.

To examine the model, we follow Bagger and Naculich [3] and consider the simple case of solitons with $N$ fermions that all appear in the lowest single particle energy state. The fermionic wave function then has the form

$$
\psi=\frac{1}{r}\left(\begin{array}{c}
i G(r) \\
F(r) \boldsymbol{\sigma} \cdot \hat{r}
\end{array}\right) \frac{\chi}{(4 \pi)^{1 / 2}}
$$

where $\chi$ is a Pauli spinor. By rescaling the scalar field to $\varphi=g \phi / \sqrt{N}$, the $N$ dependence in the Lagrangian density will contribute simply as an overall factor $N$. The effective action to one loop order in fermionic fluctuations is $\Gamma_{\text {eff }}[\varphi]=\int d^{4} x \mathcal{L}+\Gamma_{\text {vac }}[\varphi]$, where $\Gamma_{\text {vac }}[\varphi]=$ $-i \operatorname{Tr} \ln \left(S^{-1}(\varphi) S\left(\varphi_{v}\right)\right)$, and $S(\varphi)=1 /(i \not \partial-\varphi)$ is the fermionic green function operator in the background $\varphi$. This gives

$$
\Gamma_{\mathrm{vac}}[\varphi]=-i \int d^{4} x \operatorname{tr}\langle x|\ln (i \not \partial-\varphi)-\ln (i \not \partial-m)| x\rangle
$$

The ground state configuration for static fields is then the one that minimizes the energy functional $E_{\text {tot }}[\varphi]=-\Gamma_{\text {eff }}[\varphi] / \int d t$.

After some standard manipulations of Eq. (3), the vacuum energy can be written as [5,7] 


$$
E_{\mathrm{vac}}[\varphi]=-\frac{i}{2 \pi} \int d^{3} x \int d \omega \omega \operatorname{tr}\left[\gamma_{0} S(\boldsymbol{x}, \boldsymbol{x} ; i \omega)\right]
$$

where

$$
S(\boldsymbol{x}, \boldsymbol{x} ; i \omega)=\left\langle\boldsymbol{x}\left|\frac{1}{i \gamma_{0} \omega+i \boldsymbol{\gamma} \cdot \nabla-\varphi(x)}\right| \boldsymbol{x}\right\rangle
$$

is the fermion Green function in the coordinate space representation. The scalar vacuum density is obtained from the energy by taking the functional derivative $\delta E_{\text {vac }} / \delta \varphi$, giving

$$
\rho_{\mathrm{vac}}(\boldsymbol{x})=-\frac{1}{2 \pi} \int d \omega \operatorname{tr}[S(\boldsymbol{x}, \boldsymbol{x} ; i \omega)]
$$

One can derive the DE expressions for $E_{\mathrm{vac}}$ and $\rho_{\mathrm{vac}}$ by rewriting the Green function as

$$
\begin{aligned}
S(\boldsymbol{x}, \boldsymbol{x} ; i \omega) & =\int \frac{d^{3} p}{(2 \pi)^{3}}\langle\boldsymbol{x} \mid \boldsymbol{p}\rangle \frac{1}{i \gamma_{0} \omega-\boldsymbol{\gamma} \cdot \boldsymbol{p}-\varphi\left(i \nabla_{\boldsymbol{p}}\right)}\langle\boldsymbol{p} \mid \boldsymbol{x}\rangle \\
& =\int \frac{d^{3} p}{(2 \pi)^{3}} \frac{1}{i \gamma_{0} \omega-\boldsymbol{\gamma} \cdot \boldsymbol{p}-\varphi\left(\boldsymbol{x}+i \nabla_{\boldsymbol{p}}\right)},
\end{aligned}
$$

and expanding about $\varphi(x)$. Of course, the above expressions for $E_{\mathrm{vac}}$ and $\rho_{\mathrm{vac}}$ need to be regularized and renormalized to make them finite. Dimensional regularization can be used with the appropriate renormalization conditions given in [3].

It is useful to rescale all fields and variables in terms of the fermionic mass scale $m$, which we can set equal to 1 . The effective energy of the system can then be written as an energy per fermion in units of $m=g v$ :

$$
\begin{aligned}
E_{\mathrm{tot}}[\varphi] & =E_{\mathrm{scalar}}[\varphi]+\epsilon+E_{\mathrm{vac}}[\varphi], \\
E_{\text {scalar }}[\varphi] & =\frac{1}{g^{2}} \int d^{3} x\left[\frac{1}{2}(\nabla \varphi)^{2}+\frac{\mu^{2}}{8}\left(\varphi^{2}-1\right)^{2}\right], \\
E_{\mathrm{vac}}[\varphi] & =\frac{1}{16 \pi^{2}} \int d^{3} x\left[\frac{1}{2}\left(\varphi^{2}-1\right)\left(3 \varphi^{2}-1\right)-\varphi^{4} \ln \left(\varphi^{2}\right)-\ln \left(\varphi^{2}\right)(\nabla \varphi)^{2}\right]+\mathcal{O}\left(\partial^{4}\right) .
\end{aligned}
$$

The DE expression for the vacuum energy has been expanded to second order in derivatives of the scalar field [3, 11]. The fermion energy $\epsilon$ is found by solving the eigenvalue equation $(-i \gamma \cdot \nabla+\varphi) \psi=\epsilon \gamma_{0} \psi$. Minimizing the energy functional, $E_{\text {tot }}[\varphi]$, and using Eq. (2), allows us to write the field equations as 


$$
\begin{aligned}
\frac{d G}{d r} & =\frac{G}{r}+(\epsilon+\varphi) F, \\
\frac{d F}{d r} & =-\frac{F}{r}-(\epsilon-\varphi) G, \\
\nabla^{2} \varphi+\frac{\mu^{2}}{2}\left(\varphi-\varphi^{3}\right) & =g^{2}\left(\rho_{\text {val }}+\rho_{\text {vac }}\right),
\end{aligned}
$$

subject to the normalization constraint

$$
\int_{0}^{\infty} d r\left(G^{2}+F^{2}\right)=1
$$

The source terms are

$$
\begin{aligned}
\rho_{\mathrm{val}} & =\frac{1}{4 \pi r^{2}}\left(G^{2}-F^{2}\right), \\
\rho_{\mathrm{vac}} & =\rho_{\mathrm{vac}}^{\mathrm{LDA}}+\rho_{\mathrm{vac}}^{\mathrm{DE}, 2}+\mathcal{O}\left(\partial^{4}\right), \\
\rho_{\mathrm{vac}}^{\mathrm{LDA}} & =-\frac{1}{4 \pi^{2}}\left[\varphi^{3} \ln \left(\varphi^{2}\right)-\varphi^{3}+\varphi\right], \\
\rho_{\mathrm{vac}}^{\mathrm{DE}, 2} & =\frac{1}{8 \pi^{2}}\left[\frac{1}{\varphi}(\nabla \varphi)^{2}+\ln \left(\varphi^{2}\right)\left(\nabla^{2} \varphi\right)\right] .
\end{aligned}
$$

As written, these equations facilitate trying different levels of approximation to the fermionic vacuum in the self-consistent solution. Setting $\rho_{\mathrm{vac}}=0$ corresponds to the semiclassical approximation (CL), where vacuum corrections are ignored. Keeping the first term in (16) will be referred to as the local density approximation (LDA), as this term gives the exact one loop result for a spatially uniform scalar field. Including the second term in (16) will be referred to as derivative expansion (DE) approximation. The density $\rho_{\text {vac }}$ has only been expanded to second order — the same order as the differential equation (13) for $\varphi$. In our experience, attempting to include terms with higher order derivatives in self-consistent calculations tends to make the solutions numerically unstable. We discuss below a method for evaluating $\rho_{\text {vac }}$ exactly.

It was noted in [3] that for $g \rightarrow \infty$, the soliton solution satisfies a constraint that becomes independent of $g$. To see this we note that all source terms enter with the same power of $g$ in (15-18), and Eqs. (11) and (12) are $g$-independent, so with the above notation this constraint takes the simple form 


$$
\rho_{\text {val }}(r)+\rho_{\text {vac }}(r)=\mathcal{O}\left(1 / g^{2}\right) .
$$

Thus a large amount of cancellation is required between these source terms for large $g$. This restricts the form of the solutions, but does not necessarily enforce an asymptotic $g$ independent shape, as claimed in [3]. The reason is that a family of different functions may exist that satisfy Eq. (19), so that the true solution is determined by the $\mathcal{O}\left(1 / g^{2}\right)$ parts.

The field equations (11-[13) agree with those of Bagger and Naculich [3]. Although these authors checked the convergence of the DE by examining the relative size of the fourth order terms in the expansion, they did so only for the energy functional using a fixed scalar profile. The convergence, however, may be quite different for terms in the dynamical equations of motion. To see this, consider a fixed background field of the form

$$
\varphi(r)=1-\frac{a(1+f)}{e^{b r^{2}}+f e^{-b r^{2}}} .
$$

At values of $a=0.5, b=0.16$, and $f=0.8$ we obtain a scalar field that roughly corresponds to the quantum soliton solution at $g=25$ found by Bagger and Naculich [3]. Table \] shows the DE contributions to the energy and the density up to fourth order. Expressions for these fourth order contributions can be found in the Appendix. It can be seen that although the fourth order terms do not make a substantial contribution to the energy, this is not the case for the density (which is shown for $r=0$ in the table). For the self-consistent solution the convergence is worse as $g$ increases and the size of the scalar bag solution shrinks.

Our purpose therefore, is to reconsider this model and make use of the correction method described in [10] to account for the fermionic vacuum in an exact manner. This involves extending a scheme discussed by Wasson and Koonin [5.9] for calculations in one spatial dimension. In three spatial dimensions, an exact, or "brute-force" calculation involves making a partial wave expansion of the Green function (5),

$$
S\left(\boldsymbol{x}, \boldsymbol{x}^{\prime} ; i \omega\right)=\frac{1}{r r^{\prime}} \sum_{\kappa, m} S_{\kappa}\left(r, r^{\prime} ; i \omega\right) \otimes \mathcal{Y}_{\kappa m}(\hat{x}) \mathcal{Y}_{\kappa m}^{\dagger}\left(\hat{x}^{\prime}\right)
$$

Each partial wave Green function obeys the equation 


$$
\left(\begin{array}{cc}
i \omega-\varphi(r) & -\frac{d}{d r}+\frac{\kappa}{r} \\
-\frac{d}{d r}-\frac{\kappa}{r} & -i \omega-\varphi(r)
\end{array}\right) S_{\kappa}\left(r, r^{\prime} ; i \omega\right)=\delta\left(r-r^{\prime}\right)
$$

which can be solved numerically [12]. A similar partial wave expansion can be made of the DE approximation to the Green function, Eq. (7), giving $S_{\kappa}^{\mathrm{DE}}[\overline{7}$. The sum of all partial wave DE contributions to the density will reproduce the expressions (17-18).

Wasson and Koonin [5,9] pointed out that the DE approximation to the Green function works well for large loop energies $\omega$ (and similarly also for large angular momenta $\kappa$ in three spatial dimensions). Therefore one can use the DE as a sophisticated extrapolation procedure for accelerating the convergence of the brute-force method. The exact Green function contribution can be calculated up to some $\kappa_{\max }$, and the DE can be used to calculate the contribution for the remaining partial waves. An equivalent method is to use the full DE result, and correct the low energy and low partial wave terms using the difference between the exact and DE expressions for $S_{\kappa}$. This improves the convergence of the energy integral in (6). Hence we put

$$
\rho_{\mathrm{vac}}=\rho_{\mathrm{vac}}^{\mathrm{LDA}}+\rho_{\mathrm{vac}}^{\mathrm{DE}, 2}+\Delta \rho_{\mathrm{corr}}[\varphi]
$$

where the density correction is given by

$$
\begin{aligned}
\Delta \rho_{\text {corr }}[\varphi] & =\sum_{\kappa=1}^{\infty} \lim _{\Lambda_{\kappa} \rightarrow \infty} \Delta \rho_{\kappa}\left(\Lambda_{\kappa}, r\right) \\
\Delta \rho_{\kappa}\left(\Lambda_{\kappa}, r\right) & =-\frac{\kappa}{\pi^{2}} \int_{0}^{\Lambda_{\kappa}} d \omega\left[\frac{1}{r^{2}} \Re \operatorname{tr}\left[S_{\kappa}(r, r ; i \omega)\right]+\Upsilon_{\kappa}^{1} \varphi-\frac{1}{2} \Upsilon_{\kappa}^{2}\left(\nabla^{2} \varphi\right)\right. \\
& \left.\quad-\frac{2}{3} \Upsilon_{\kappa}^{3} \varphi^{2}\left(\nabla^{2} \varphi\right)-\frac{5}{3} \Upsilon_{\kappa}^{3} \varphi(\nabla \varphi)^{2}-2 \Upsilon_{\kappa}^{4} \varphi^{3}(\nabla \varphi)^{2}\right]
\end{aligned}
$$

where

$$
\begin{aligned}
& \Upsilon_{\kappa}^{n}(r)=\frac{1}{4 \pi r^{2}}\left[\Delta_{\kappa-1}^{n}(r)+\Delta_{\kappa}^{n}(r)\right] \\
& \Delta_{\kappa}^{n}(r)=-\frac{1}{(n-1) !}\left(\frac{1}{2 z_{\omega}} \frac{d}{d z_{\omega}}\right)^{n-1}\left[z_{\omega} r^{2} i_{\kappa}\left(z_{\omega} r\right) k_{\kappa}\left(z_{\omega} r\right)\right],
\end{aligned}
$$

$z_{\omega}=\left(\omega^{2}+\varphi^{2}\right)^{1 / 2}, \varphi=\varphi(r)$, and $i_{\kappa}, k_{\kappa}$ are the modified spherical Bessel functions of order $\kappa$. The utility of this method is that one only needs to include in $\Delta \rho_{\text {corr }}$ as many partial 
waves as are needed to achieve the desired accuracy. Also, $\Delta \rho_{\text {corr }}$ is a finite quantity that is independent of renormalization. The renormalization counterterms only appear in the DE expressions (17-18). The details of deriving this correction are described in [10].

Numerically, it is useful to be able to treat our equations entirely as a boundary value problem. This can be done by treating $\epsilon$ as a field, and also introducing a field $\chi$ for the auxiliary equation (14), so that

$$
\frac{d \epsilon}{d r}=0, \quad \frac{d \chi}{d r}=G^{2}+F^{2}
$$

The boundary conditions for the entire system of equations are then

$$
\begin{aligned}
\left.\frac{d \varphi(r)}{d r}\right|_{r \rightarrow 0} & =0, & \left.\frac{d \varphi(r)}{d r}\right|_{r \rightarrow R} & =\left(\mu+\frac{1}{R}\right)[1-\varphi(R)], \\
\left.\frac{F(r)}{r G(r)}\right|_{r \rightarrow 0} & =\frac{1}{3}[\varphi(0)-\epsilon], & \frac{F(R)}{G(R)} & =-\left[\frac{\varphi(R)-\epsilon}{\varphi(R)+\epsilon}\right]^{1 / 2}, \\
\chi(0) & =0, & \chi(R) & =1,
\end{aligned}
$$

where $R$ is large compared to the length scale of the problem. To solve our equations we make use of the program COLNEW by Ascher and Bader [13. Unfortunately, when the correction (25) is included we cannot simply use the COLNEW routine as written, since our equations are actually integro-differential equations. To facilitate this, COLNEW was modified so that the scalar field solution and its derivative can be extracted at intermediate stages of the calculation to evaluate the density correction. The calculation is then internally iterative, with $\Delta \rho_{\text {corr }}$ treated as a source term that is reevaluated as needed with each internal iteration of the COLNEW code.

We now examine the results when using different levels of approximation to the vacuum. We use the schemes discussed above in the following order: CL, LDA, DE, and EX. Here EX will refer to the exact solution, which includes $\Delta \rho_{\text {corr }}$ (24). Our goal is to quantify the effect of this correction on the self-consistent solution for various couplings. The choice $g=10$, $\mu=1 / 10$ will serve as an example. (As the energy scale $m$ already includes an implicit dependence on $g$, setting $\mu=1 / g$ simply fixes the scalar field mass at the VEV value.) 
For the CL, LDA, and DE approximations the self-consistent solutions for $\varphi$ are shown in Fig. 1. We see that adding the LDA term reduces the depth and width of the scalar field, so that a zero no longer appears. The total energy of the solution, $E_{\text {tot }}=.960 m(\mathrm{LDA})$, has dramatically changed from that of the semi-classical approximation, $E_{\text {tot }}=.620 m(\mathrm{CL})$. This behavior, which was identified by [3] at the DE level, occurs even before the derivative terms are included. However, for $g>10.4$ the LDA approximation gives no solution. Looking at the expression for the LDA contribution in (17), we see that this function is odd with respect to the scalar field. Increasing the coupling drives the scalar field deeper, so that near $g=10.4$ the solution becomes negative at $r=0$, and the LDA contributes with the opposite sign. At this point the equations no longer support a solution as it is impossible to satisfy the constraint (19). However, when the DE terms are included the vacuum density is not forced to change sign as $g$ is increased, and a soliton solution exists. In Fig. 1 we see that the shape of the scalar field for $g=10$ is even shallower for the DE than for the LDA. An interesting feature to note is that under the DE the total energy, $E_{\text {tot }}=.967 m(\mathrm{DE})$, is relatively unchanged from the LDA result. This occurs even though the Fermi level has changed from $\epsilon=.863 m(\mathrm{LDA})$ to $\epsilon=.908 m(\mathrm{DE})$, and is made possible due to a corresponding decrease in the energy contribution from the scalar field, $E_{\text {scalar }}+E_{\mathrm{vac}}$.

Now examine what happens when the density correction terms are included. In Fig. 2 the self-consistent scalar field solutions are shown for calculations including an increasing number of partial wave corrections. The exact vacuum density favors a deeper scalar field than the DE. The fifth partial wave has not been included in the figure since the field in this case is found to be indistinguishable from that with four partial waves. Looking at the sequence of scalar fields in Figs. 1 and 2 we see a manifestation of the fact that terms in the derivative expansion display oscillatory convergence [12]. Note that after including the $\kappa=1$ term in the correction (25) the correct vacuum density is specified at the origin as higher terms are zero there. However, the $\kappa>1$ terms may still affect the self-consistent solution. In fact, we see in Fig. 2 that the scalar field becomes deeper as subsequent terms in the correction are included. The corrected solution gives $\epsilon=.857 \mathrm{~m}(\mathrm{EX})$, and $E_{\mathrm{tot}}=.976 \mathrm{~m}$ 
(EX). The energy of the scalar field is twice the DE result, and the Fermi level has dropped away from $m$. However, the total energy remains fairly stable, rising only slightly above the DE result.

To get the EX correction it is sufficient to use partial waves up to $\kappa=4$, except at large $g$ where $\kappa=5$ is needed. In Fig. 3 we see that for other values of $g$ there is also a decrease in the depth of the scalar field using the exact calculation. The exact results agree with the DE calculation for small coupling as expected. As $g$ is increased the depth of the bag decreases. For $g>20$ values of the wave function $\varphi(r)$ near $r=0$ are numerically uncertain by about 0.02 . This uncertainty is a symptom of the constraint (19), but in no way affects the value of the energies. In Fig. 1 we show how including the correction affects the energies at different $g$. ¿From the fermion energy, $\epsilon$, we see that the corrections affect the solution for all $g>5$. As the coupling increases we have found that not only does the width of the bag shrink, but the depth increases. Since this leads to larger derivatives, the energy of the scalar field increases. Correspondingly, fermions may be more tightly bound in the bag so that the Fermi energy level decreases. When the fermionic and scalar field energies are added to obtain the total energy, the sum gives an answer that is closer to the DE result than either of the components separately. This occurs at all $g$. In fact, it is surprising to find that the corrected total energy remains fairly constant as the coupling is increased, exhibiting nearly the same scaling as the DE total energy. Recall that our energy is scaled in terms of the fermionic mass $m=g v$, so that relative to $v$ the energy scales directly proportional to the coupling. Thus the claim that such scaling behavior is universal [14] is supported by our calculations.

In summary, we have found a way of correcting the DE approximation for fermionic vacuum fluctuations while still demanding a self-consistent solution. There remain several ways in which the results of the calculation done here may be made more general. To include different fermionic energy levels it would likely be more economical to use the COLNEW routine to solve only the scalar equation, while solving the eigenvalue problem for the relevant fermionic wave function components using standard Runge-Kutta techniques. If one takes 
for granted some validity in the one loop approximation for finite $N$ and large coupling, it should also be possible to include the scalar fluctuations at the one loop level. It is expected that these fluctuations would contribute a source density term with the opposite sign to the fermionic fluctuations [7]. However, it is likely that a similar density correction calculation would be necessary for the scalar loops to ascertain that this contribution to the true scalar vacuum was also included correctly.

\section{ACKNOWLEDGMENTS}

One of us (P.G.B.) would like to thank the Institute for Nuclear Theory and the Nuclear Theory group at the University of Washington for their hospitality and support during his sabbatical leave. I.W.S. would like to thank Martin Gremm, Anton Kapustin, and Mark Wise for their comments. This work was also supported in part by the Natural Sciences and Engineering Research Council of Canada, and by the U. S. Department of Energy under grant DE-FG03-92-ER40701.

\section{APPENDIX A: FOURTH ORDER DERIVATIVE EXPANSION OF THE ENERGY AND DENSITY}

The expressions for the energy and density at fourth order, which were used in Table 凹, are

$$
\begin{aligned}
E_{\mathrm{vac}}[\varphi]= & -\frac{1}{160 \pi^{2}} \int d^{3} x\left[\frac{\left(\partial^{2} \varphi\right)^{2}}{\varphi^{2}}-\frac{11\left(\partial_{\alpha} \varphi\right)^{2}\left(\partial^{2} \varphi\right)}{9 \varphi^{3}}+\frac{11\left(\partial_{\alpha} \varphi\right)^{4}}{18 \varphi^{4}}\right] \\
\rho_{\mathrm{vac}}^{D E, 4}= & -\frac{1}{80 \pi^{2} \varphi^{2}}\left[\partial^{4} \varphi-\frac{25\left(\partial_{\alpha} \partial^{2} \varphi\right)\left(\partial^{\alpha} \varphi\right)}{9 \varphi}-\frac{11\left(\partial^{2} \partial_{\alpha} \varphi\right)\left(\partial^{\alpha} \varphi\right)}{9 \varphi}-\frac{16\left(\partial^{2} \varphi\right)^{2}}{9 \varphi}\right. \\
& \left.-\frac{11\left(\partial_{\alpha} \partial_{\beta} \varphi\right)^{2}}{9 \varphi}+\frac{43\left(\partial_{\alpha} \varphi\right)^{2}\left(\partial^{2} \varphi\right)}{9 \varphi^{2}}+\frac{44\left(\partial_{\alpha} \partial_{\beta} \varphi\right)\left(\partial^{\alpha} \varphi\right)\left(\partial^{\beta} \varphi\right)}{\left.9 \varphi^{2}\right)}-\frac{11\left(\partial_{\alpha} \varphi\right)^{4}}{3 \varphi^{3}}\right] .
\end{aligned}
$$

Covariant notation is used for convenience. 


\section{REFERENCES}

[1] R. J. Perry, Phys. Lett. B 182, 269 (1986); R. J. Perry, ibid. 214, 307 (1986); D. A. Wasson, ibid. 210, 41 (1988); W. R. Fox, Nucl. Phys A495, 463 (1989); R. J. Furnstahl and C. E. Price, Phys. Rev. C 41, 1792 (1990); R. J. Furnstahl, H. Tang, and B. D. Serot, ibid. 52, 1368 (1995).

[2] P. Gusynin and I. A. Shovkovy, University of Groningen Report No. UG-9/95, hepph/9509383, 1995; D. Cangemi, E. D’Hoker, and G. Dunne, Phys. Rev. D 51, 2513 (1995).

[3] J. Bagger and S. Naculich, Phys. Rev. Lett. 67, 2252 (1991); J. Bagger and S. Naculich, Phys. Rev. D 45, 1395 (1992).

[4] R. J. Perry, Nucl. Phys. A467, 717 (1987).

[5] D. A. Wasson, Nucl. Phys. A535, 456 (1991).

[6] M. Li, R. J. Perry, and L. Wilets, Phys. Rev. D 36, 596 (1987).

[7] M. Li and R. J. Perry, Phys. Rev. D 37, 1670 (1988).

[8] P. G. Blunden, Phys. Rev. C 41, 1851 (1989).

[9] D. A. Wasson and S. Koonin, Phys. Rev. D 43, 3400 (1991).

[10] I. W. Stewart, M.Sc. thesis, University of Manitoba, 1995; I. W. Stewart and P. G. Blunden, 1996 (in preparation).

[11] L. H. Chan, Phys. Rev. Lett. 54, 1222 (1985).

[12] M. Li, L. Wilets, and R. J. Perry, J. Comput. Phys. 85, 457 (1989).

[13] G. Bader and U. Ascher, Siam J. Scient. Stat. Comput. 8, 483 (1987).

[14] S. Naculich, Phys. Rev. D 46, 5487 (1992). 


\section{TABLES}

TABLE I. Contributions from different orders of the DE series for the energy and the density. Results are shown for a fixed background field $\varphi(r)$ with $a=0.5, b=0.16$, and $f=0.8$. Units are given in terms of the scale $m$, as indicated.

\begin{tabular}{lcrr}
\hline \hline & LDA & 2nd order & 4th order \\
\hline$E_{\text {vac }}\left(10^{-2} m\right)$ & 5.909 & 2.001 & -0.636 \\
$\rho_{\text {vac }}(r=0)\left(10^{-3} m^{3}\right)$ & -5.109 & -0.936 & -3.732 \\
\hline \hline
\end{tabular}




\section{FIGURES}

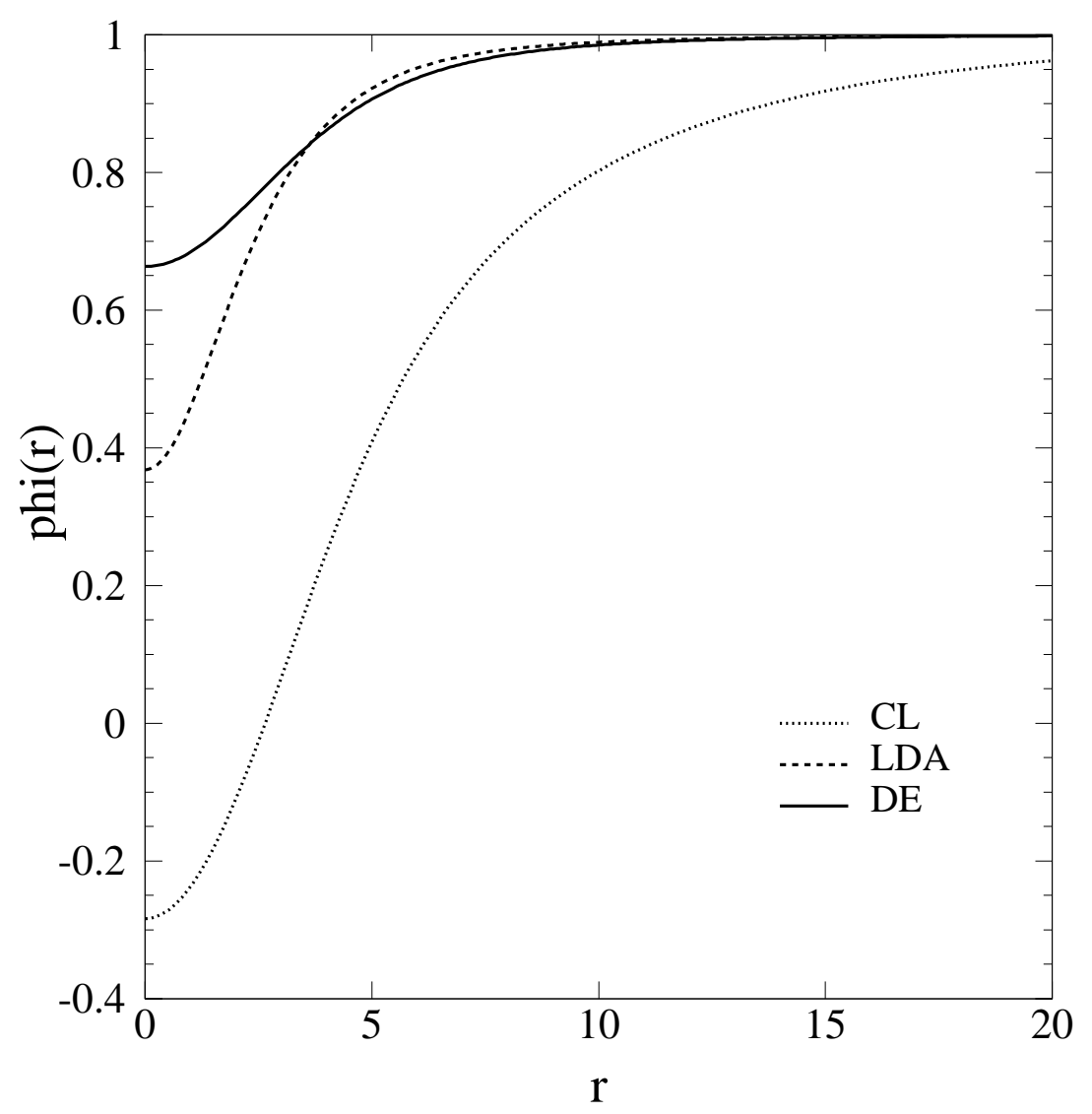

FIG. 1. Self-consistent scalar field solutions of the Bagger-Naculich model $(g=10, \mu=1 / 10)$ for semi-classical (CL), local density (LDA), and derivative expansion (DE) approximations to the vacuum densities. 


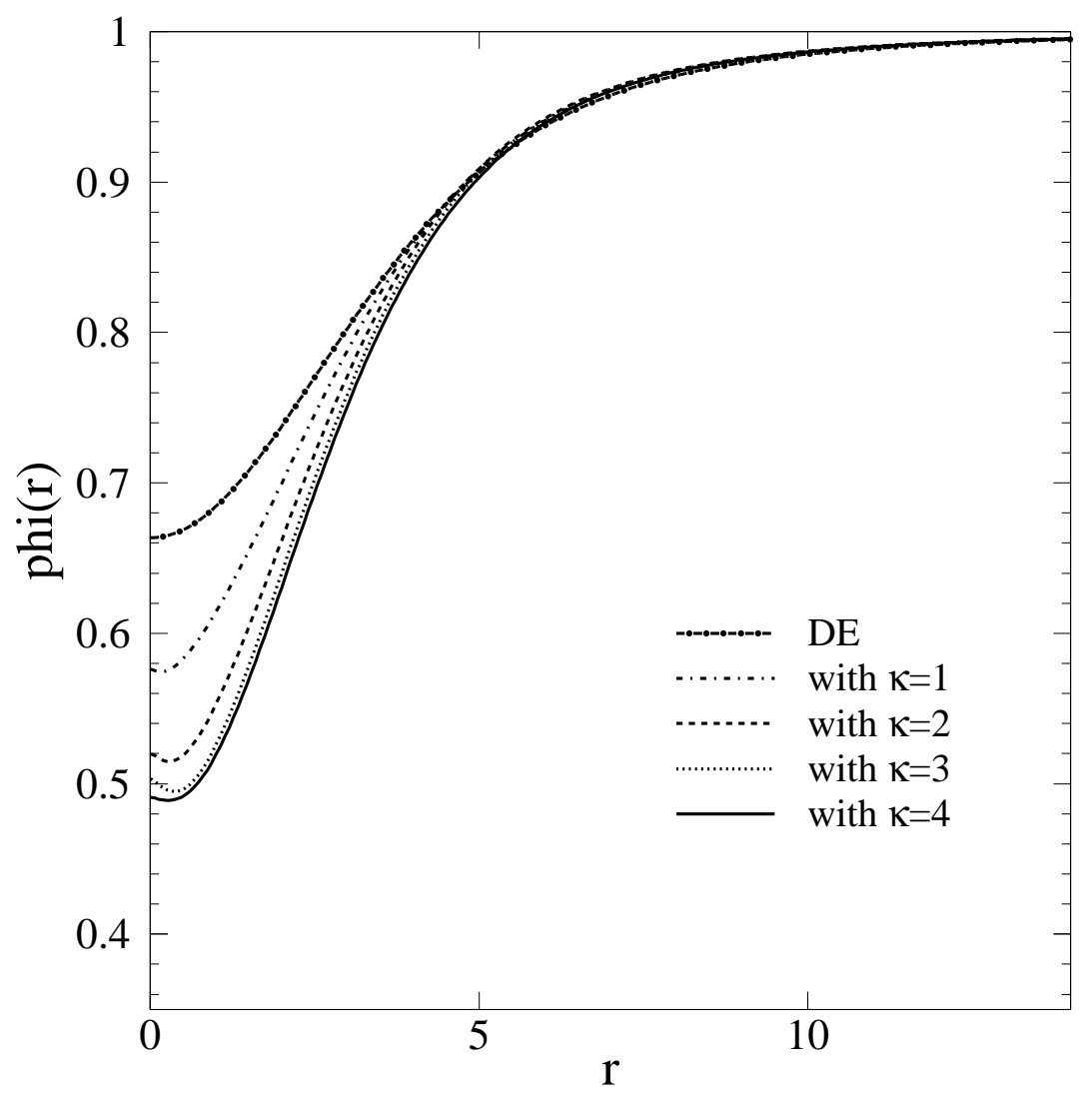

FIG. 2. Self-consistent scalar field solutions with increasing number of terms in the partial wave correction series. $g=10, \mu=1 / 10$. 


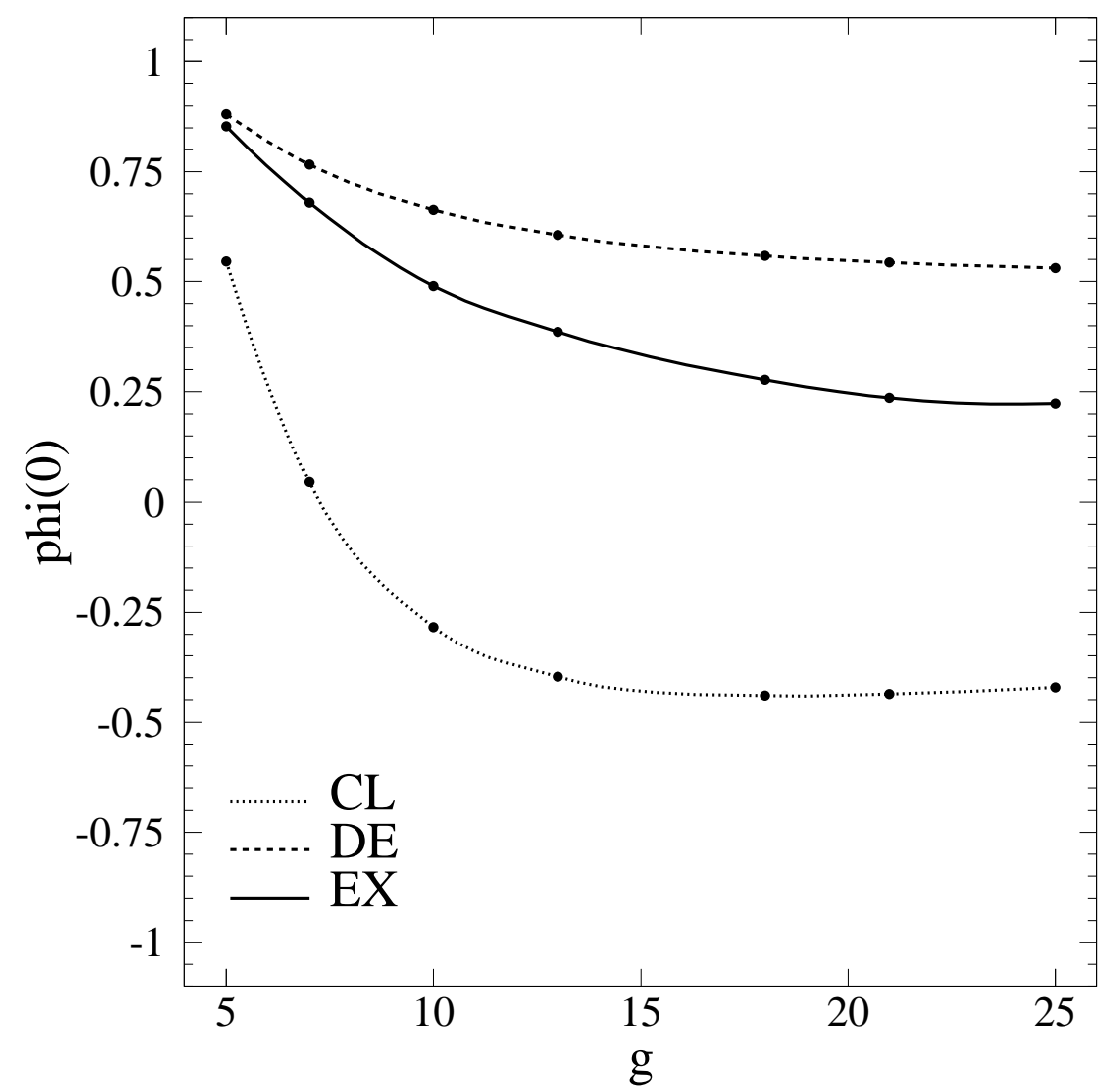

FIG. 3. Depth of the self-consistent scalar field solutions $\varphi(r=0)$ for different values of the coupling $g$, and $\mu=1 / g$. Results are shown for the semi-classical (CL), derivative expansion (DE), and exact (EX) solutions. 


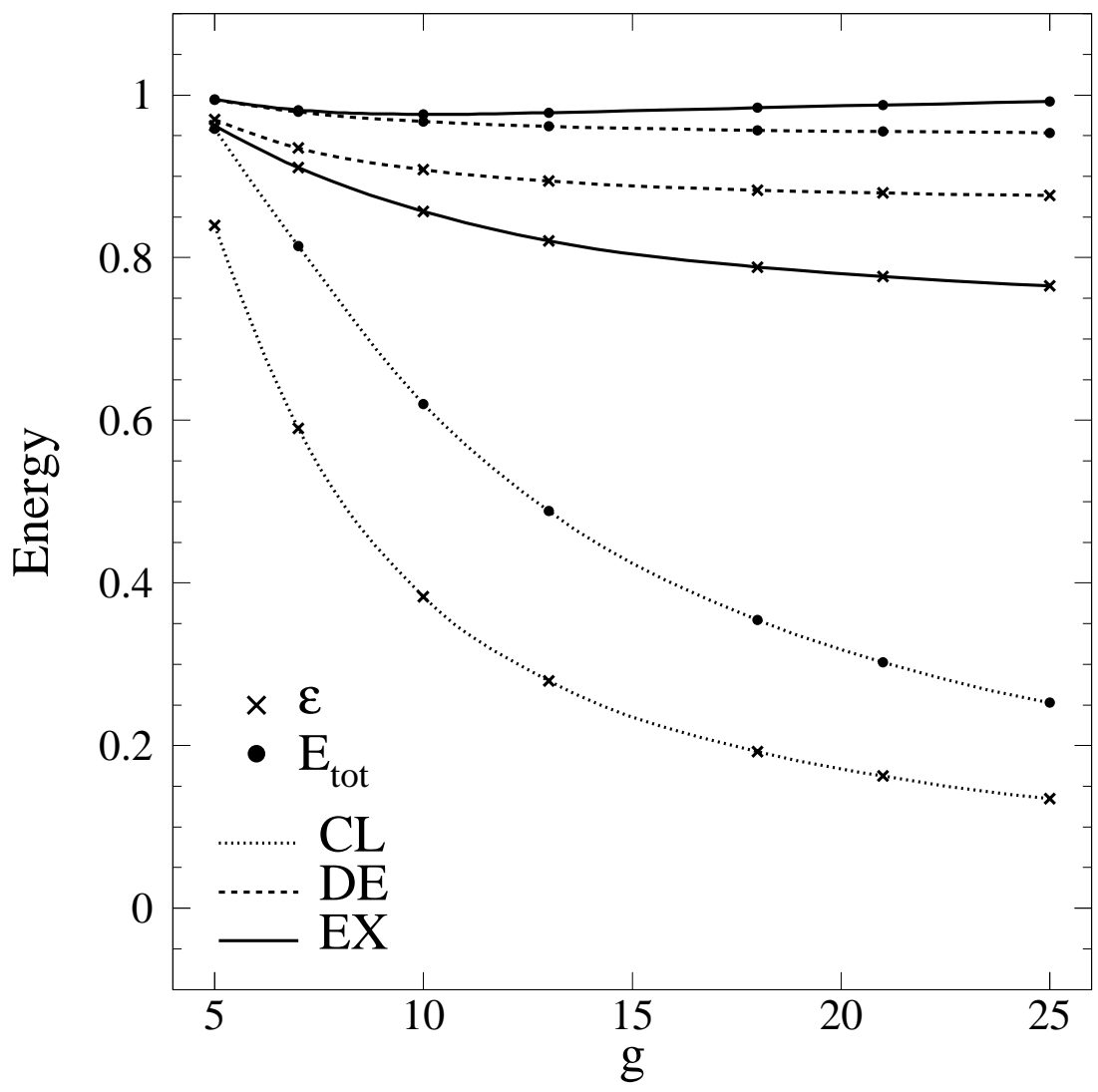

FIG. 4. Self-consistent fermion $(\epsilon)$ and total soliton $\left(E_{\text {tot }}\right)$ energies for different values of the coupling $g$, with $\mu=1 / g$. 